\title{
Effect of the C(3)-Substituent in Verdazyl Radicals on their Profluorescent Behavior
}

\author{
Steffen Eusterwiemann ${ }^{a}$, David Matuschek ${ }^{a}$, Linda Stegemann ${ }^{b}$, Sina Klabunde ${ }^{c}$, \\ Carsten Doerenkamp ${ }^{c}$, Constantin G. Daniliuc ${ }^{a}$, Nikos L. Doltsinis ${ }^{\star d}$, Cristian A. Strassert ${ }^{\star b}$, \\ Hellmut Eckert ${ }^{\star c e}$, and Armido Studer ${ }^{\star a}$
}

\begin{abstract}
Methods for the detection of reactive intermediates such as transient radicals are important in organic chemistry, polymer chemistry, biology or medicine. Along these lines we recently reported that 1,5-diphenyl-6oxo verdazyl radicals can be used as fluorescent spin sensors. In situ generated C-centered radicals are efficiently trapped by the verdazyls, which in turn undergo transformation from a paramagnetic non-fluorescent state to a diamagnetic fluorescent state. Whereas the $N$-phenyl substituent in the spin probes is of high importance for obtaining profluorescent behavior, the effect of the $\mathrm{C}(3)$-substituent has not been investigated to date. We herein present the synthesis and characterization of various 1,5-diphenyl-6-oxo-verdazyl radicals bearing differently hybridized C-substituents at the C(3) position. Steady-state and time-resolved fluorescence spectroscopy in solution and in the solid state along with time-dependent density functional theory (TDDFT) calculations reveal that a C(3)-aryl substituent is crucial for obtaining fluorescence after spin trapping. In addition, it is shown that the emission wavelength of the $\mathrm{C}(3)$-aryl substituted verdazyl derivatives can be tuned by selective destabilization of the HOMO and the LUMO.
\end{abstract}

Keywords: EPR · Profluorescence · Spin traps · TDDFT · Verdazyl radicals

\section{Introduction}

1,5-Diphenyl-6-oxo verdazyls are persistent radicals, ${ }^{[1]}$ which have been used as building blocks for magnetic materials, ${ }^{[2]}$ as substrates for organic synthesis, ${ }^{[3]} \mathrm{ESR}$ spin labels ${ }^{[4]}$ and as mediators in living radical polymerizations. ${ }^{[5]}$ Recently, we reported their profluorescent behavior, allowing these paramagnetic species to be used as spin sensors. ${ }^{[6]}$ Fluorescence is fully suppressed in these paramagnetic

${ }^{*}$ Correspondence: Prof. N. L. Doltsinis ${ }^{d}$, Prof. Dr. H. Eckert ${ }^{\mathrm{ce}}$, PD Dr. C. Strassert ${ }^{\mathrm{b}}$, Prof. Dr. A. Studer E-Mail: nikos.doltsinis@uni-muenster.de, eckerth@uni-muenster.de, ca.s@uni-muenster.de, studer@uni-muenster.de

alnstitute of Organic Chemistry, Department of Chemistry and Pharmacy, Westfälische Wilhelms-Universität Münster, Corrensstrasse 40, 48149 Münster, Germany ${ }^{\mathrm{b}}$ Center for Nanotechnology, Institute of Physics, Westfälische Wilhelms-Universität Münster, Heisenbergstrasse 11, 48149 Münster, Germany. E-mail: ca.s@uni-muenster.de.

Institut für Physikalische Chemie, Westfälische

Wilhelms-Universität Münster, Corrensstrasse 28/30, 48149 Münster, Germany

dInstitut für Festkörpertheorie and Center for Multi-

scale Theory \& Computation, Westfälische Wilhelms-

Universität Münster, Wilhelm-Klemm-Strasse 10,

48149 Münster, Germany

eInstituto da Física em Sao Carlos, Universidade de

Sao Paulo, Avenida Trabalhador Saocarlense 400,

Sao Carlos, SP 13560, Brazil species due to quenching of the excited state, whereas upon reaction with transient radicals the fluorescence is restored in the corresponding diamagnetic spin trapped products (Fig. 1). Since transient radicals are common intermediates in organic synthesis, polymer, biological and environmental chemistry, methods for the detection of these reactive intermediates are important. [7] Prior to our report, this principle was applied to nitroxide radicals bearing a fluorophor covalently connected to the nitroxyl moiety via a short tether. ${ }^{[8]}$ Along with their application as spin labels, ${ }^{[9]}$ the profluorescent behavior of nitroxides was also exploited to image cellular oxidative stress $^{[10]}$ and polypropylene degradation. ${ }^{[11]}$ In contrast, oxo-verdazyl radicals do not require covalent attachment of a fluorophor, since these heterocycles in a paramagnetic form show intrinsic fluorescence properties. Our studies revealed that $N$-phenyl substituents in verdazyl radicals

are essential for obtaining profluorescent behavior. Diamagnetic spin trapping products derived from $\mathrm{N}$-alkyl verdazyl radicals are non-fluorescent. ${ }^{[6]}$ However, the effect of the $\mathrm{C}(3)$-substituent in verdazyls on their profluorescent properties has not been investigated to date.

Herein we report the synthesis and characterization of various $\mathrm{C}(3)$-substituted 1,5-diphenyl-6-oxo verdazyl radicals and address their profluorescent behavior by studying fluorescence after trapping with C-radicals. Along these lines, we will investigate the simple $\mathrm{C}(3)-\mathrm{H}$ derivative and systems bearing differently hybridized C-substituents ( $\mathrm{sp}, \mathrm{sp}^{2}$ and $\left.\mathrm{sp}^{3}\right)$ at the $\mathrm{C}(3)$ position of the heterocycle. In addition, we will show how the physicochemical properties of such heterocycles can be tuned by introducing electron withdrawing and electron donating groups at the paraposition of phenyl substituents at position $\mathrm{N}(1),(\mathrm{N} 5)$ and $\mathrm{C}(3)$.

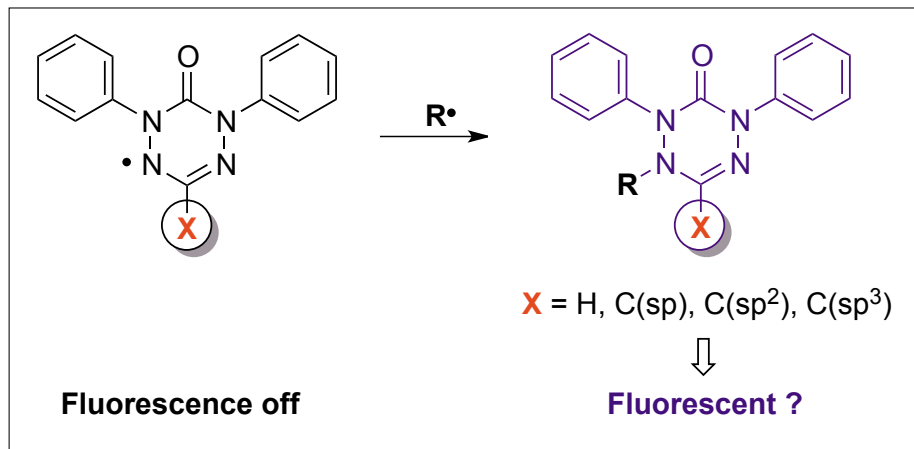

Fig. 1. Profluorescent verdazyl radicals. 


\section{Results and Discussion}

Verdazyl radicals were prepared in two steps via first condensing carbonohydrazide $\mathbf{1 a}$ or $\mathbf{1 b}$ with aldehydes $\mathbf{2}$ to afford tetrazinanones $\mathbf{3 a}-\mathbf{3 m}$ (Table 1, for details, see ESI). ${ }^{[12]}$ Oxidation with 1,4-benzoquinone gave the corresponding verdazyl radicals $\mathbf{4 a}-\mathbf{4 m}$ in good to excellent yields. Atom transfer coupling reaction ${ }^{[13]}$ of styryl bromide with the verdazyl radicals provided the trapping products $\mathbf{5 a}-\mathbf{5} \mathbf{l}$ in excellent yields. However, a nitro-substituted verdazyl radical $\mathbf{4 m}$ could not be transformed to the desired product under these conditions.
The verdazyl radicals $\mathbf{4 a - 4 m}$ were analyzed in solution by UV/Vis and EPR spectroscopy and for radical $4 \mathbf{i}$ also by X-ray structure analysis (Fig. 2).

The UV/Vis spectra of these radicals (see ESI, Fig. S7-S10) show a band, which depends strongly on the substituent. Systems with $\mathrm{H}$ and alkyl substituents at C(3) (4a: $481 \mathrm{~nm}$; 4b: $497 \mathrm{~nm}$; 4c: 495 nm; 4d: $495 \mathrm{~nm}$; 4 e: $490 \mathrm{~nm}$ ) show an absorption maximum from $481-497 \mathrm{~nm}$. In contrast, all other verdazyl radicals bearing a $\pi$-system at the $\mathrm{C}(3)$ position show bathochromic absorption behavior ranging from $533-663 \mathrm{~nm}$. In addition, the results illustrate that the absorption maxima of systems with electron withdrawing sub-

Table 1. Synthesis of verdazyl radicals 4 and trapping with C-centered radicals to $\mathbf{5}$.
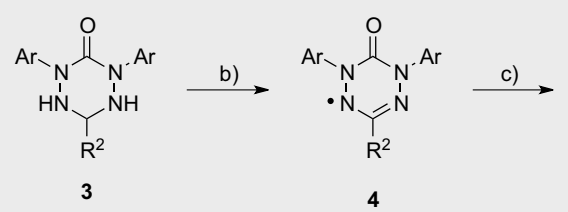

4

3 (yield)

3a $(64 \%)$

$3 \mathbf{b}(62 \%)$

3 c $(61 \%)$

3d $(73 \%)$

3e $(86 \%)$

3f $(68 \%)$

3g (50\%)

3h $(67 \%)$

3i $(68 \%)$

3j $(63 \%)$

3k $(30 \%)$

3l $(42 \%)$

3m $(76 \%)$
4 (yield)

4a $(98 \%)$

4b $(89 \%)$

4c $(64 \%)$

4d $(91 \%)$

4e $(55 \%)$

4f $(98 \%)$

4g $(85 \%)$

4h $(89 \%)$

4i $(88 \%)$

4j (99\%)

4k $(94 \%)$

$4 \mathbf{l}(89 \%)$

4m $(45 \%)$

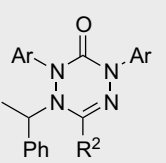

5

$13 \mathrm{H} \quad p-\mathrm{NO}_{2}-\mathrm{C}_{6} \mathrm{H}_{4} \quad 3 \mathbf{3 m}(76 \%) \quad \mathbf{4 m}(45 \%) \quad$ -

a) $\mathrm{R}^{2}-\mathrm{CHO}$ (2) (1.0 equiv.), $\mathrm{MeOH}$, reflux; b) 1,4-benzoquinone (1.7 equiv.), $\mathrm{CH}_{2} \mathrm{Cl}_{2}$, sealed tube, $60{ }^{\circ} \mathrm{C}, 2-3 \mathrm{~h}$; c) 1-bromoethylbenzene (1.2-1.5 equiv.), Cu (1.2-1.5 equiv.), Cu(OTf) $(3-5$ mol\%), 4,4'-di-tert-butyl-2,2'-dipyridyl (6-10 mol\%), $\mathrm{PhH}, 80^{\circ} \mathrm{C}, 3-6 \mathrm{~h}$.

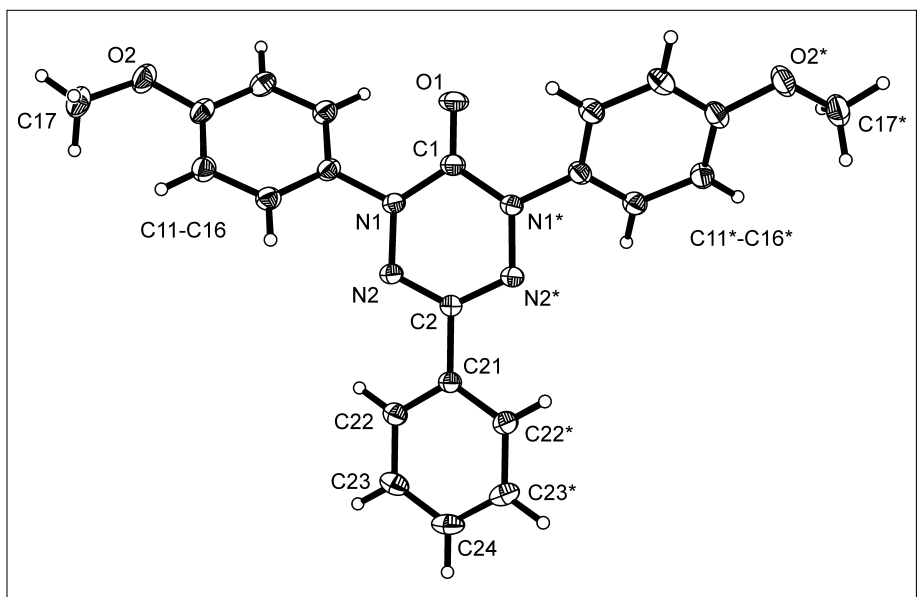

stituents in the para-position of the $\mathrm{C}(3)$ aryl ring like $-\mathrm{NO}_{2}(4 \mathrm{~m}: 533 \mathrm{~nm})$ or $-\mathrm{F}(4 \mathrm{l}$ : $559 \mathrm{~nm})$ appear blue shifted compared to the red shifted absorptions of systems with electron donating groups like -OMe (4j: $581 \mathrm{~nm})$ or $-\mathrm{NMe}_{2}(4 \mathrm{k}: 663 \mathrm{~nm})$.

The X-band EPR spectra (Fig. 3 and Table 2) recorded on $1 \mathrm{mM}$ solutions in degassed $\mathrm{CH}_{2} \mathrm{Cl}_{2}$ of all the radicals are dominated by the magnetic hyperfine interaction with the ${ }^{14} \mathrm{~N}$ nuclei $(\mathrm{N}-1,5$ and $\mathrm{N}-2,4$; quintet of quintets). As generally found in the literature, ${ }^{[14]} \mathrm{A}(\mathrm{N}-1,5)$ is significantly smaller than $\mathrm{A}(\mathrm{N}-2,4)$. In addition, super-hyperfine coupling of the unpaired electron to the hydrogen of the different substituents via spin polarization can be resolved in some cases: For compounds $4 \mathbf{a}-\mathbf{4 d}$, and $\mathbf{4 g}$ improved fits to the experimental spectra can be obtained by considering the effect of the ${ }^{1} \mathrm{H}$ nuclei associated with the N-phenyl substituents in ortho-, meta- and para-position (4:4:2 ratio). For the other compounds, hyperfine couplings with these aromatic protons were also considered, however, generally found to be near or below $0.5 \mathrm{G}$. For compound $4 \mathbf{a}$ the hyperfine coupling constant of the ${ }^{1} \mathrm{H}$ nuclei attached to $\mathrm{C}(3)$ is too small to be detected. The modest values of these proton super-hyperfine coupling constants (with the exception of compounds $\mathbf{4 b}$ and 4c), show that the radical density is concentrated on the constituent atoms of the verdazyl ring.

In comparison to the colored verdazyl radicals the diamagnetic trapping products 5 are all colorless solids, which were in some cases characterized in the solid state by single-crystal X-ray crystallography (Fig. 4 and Fig. 5). Single crystals of $\mathbf{5 b}$, $\mathbf{5 e ,}, \mathbf{5 i}$ and $\mathbf{5 l}$ were obtained by slow evaporation of a solution of the sample in EtOAc or $\mathrm{CHCl}_{3}$. In contrast to the almost planar heteroarene in the X-ray crystal structure of verdazyl radical $4 \mathbf{i}$ (CNNC dihedral angle $\left.=-1.5(1)^{\circ}\right)$, the hetero core of $5 \mathbf{i}$ appears distorted with a dihedral angle of $-25.7(3)^{\circ}$, which was also found for other derivatives of the trapping products ${ }^{[6]}$ (CNNC dihedral angles for 5b: $31.0(4)^{\circ}$, 5e: $33.9(3)^{\circ}$, 5l: $34.8(2)^{\circ}$ ) (for more details and X-ray crystal structures of $\mathbf{5 e}$ and $\mathbf{5 l}$, see ESI, Fig. S3 - S6).

The fluorescence properties of the trapping products 5 were analyzed by UV/Vis, steady-state and time-resolved fluorescence spectroscopy in solution and in the solid state (Table 3 and Fig. 6, for more details, see ESI, Fig. S11 - S61).

The absorption spectra (Fig. 6) show a band around $260 \mathrm{~nm}$ for all trapping products, except for $\mathbf{5 k}$, which appears 20 $\mathrm{nm}$ red-shifted. Upon substitution with an alkenyl, alkynyl or phenyl group $(\mathbf{5 f}-\mathbf{5 l}$, 5n), a new absorption band around 330 $\mathrm{nm}$ appears, due to electronic delocaliza- 


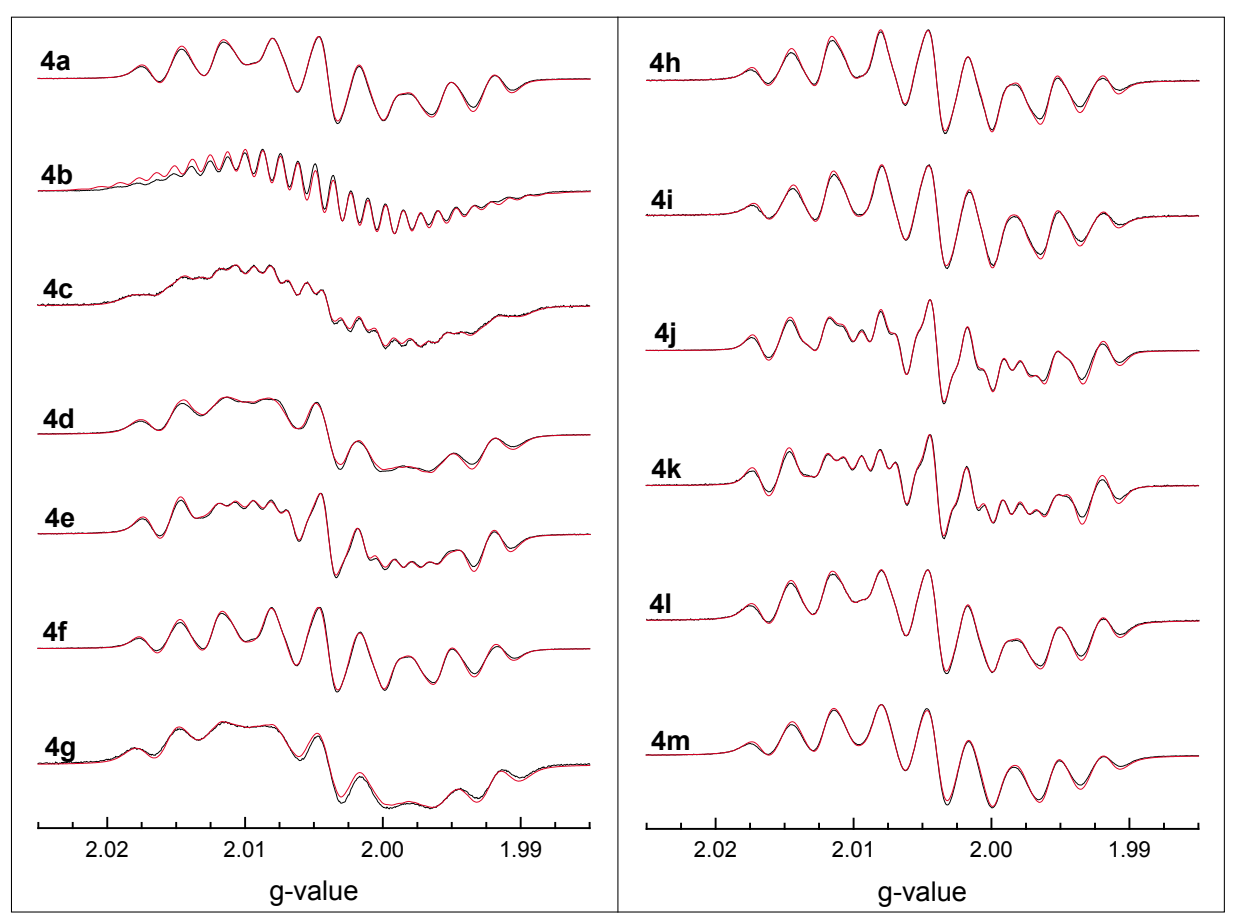

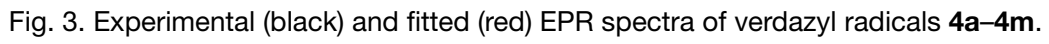

Table 2. EPR parameters (hyperfine coupling constants (in G)) and g-values for verdazyl radicals 4a-4m. Simulations include the effects of the modulation amplitudes and an additional Lorentzian line broadening parameter of $0.1 \mathrm{G}$.

\begin{tabular}{|l|c|c|c|c|c|c|c|}
\hline & $\mathbf{A}$ & $\mathrm{A}$ & $\mathrm{A}$ & $\mathrm{A}$ & $\mathrm{A}$ & $\mathrm{A}$ & $\mathrm{g}$-value \\
\hline $\mathbf{4 a}$ & 6.467 & 4.575 & 0.7 & 0.6 & $<0.5$ & $<0.5$ & 2.00394 \\
\hline $\mathbf{4 b}$ & 6.451 & 4.439 & 2.55 & 2.40 & 0.8 & & 2.00452 \\
\hline $\mathbf{4 c}$ & 6.546 & 4.526 & 1.69 & 0.7 & 0.6 & & 2.00394 \\
\hline $\mathbf{4 d}$ & 6.533 & 4.436 & 1.13 & 0.5 & $<0.5$ & 2.00395 \\
\hline $\mathbf{4 e}$ & 6.583 & 4.419 & 0.7 & 0.5 & $<0.5$ & \\
\hline $\mathbf{4 f}$ & 6.508 & 4.684 & 0.7 & 0.6 & $<0.5$ & 2.00395 \\
\hline $\mathbf{4 g}$ & 6.528 & 4.434 & 0.9 & 0.8 & $<0.5$ & & 2.00396 \\
\hline $\mathbf{4 h}$ & 6.350 & 4.665 & 0.5 & $<0.5$ & & 2.00388 \\
\hline $\mathbf{4 i}$ & 6.254 & 4.784 & $<0.5$ & $<0.5$ & & 2.00395 \\
\hline $\mathbf{4 j}$ & 6.513 & 4.525 & 0.6 & 0.6 & $<0.5$ & & 2.00389 \\
\hline $\mathbf{4 k}$ & 6.552 & 4.439 & 0.6 & 0.5 & $<0.5$ & & 2.00391 \\
\hline $\mathbf{4 l}$ & 6.438 & 4.572 & 0.6 & 0.6 & $<0.5$ & & 2.00395 \\
\hline $\mathbf{4 m}$ & 6.274 & 4.720 & & & & 2.00392 \\
\hline
\end{tabular}

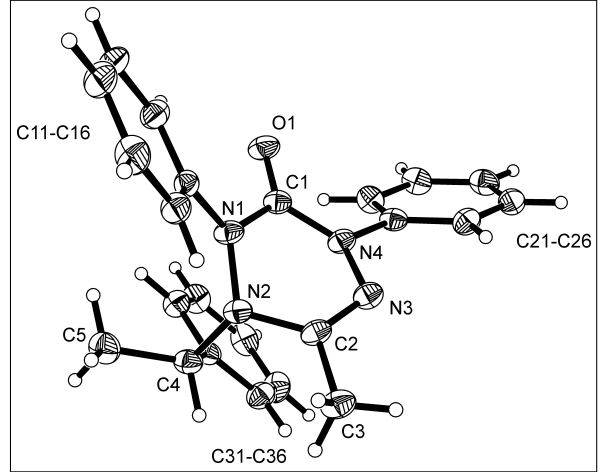

Fig. 4. X-ray crystal structure analysis of methyl-substituted trapping product $\mathbf{5 b}$ (thermal ellipsoids are shown with $30 \%$ probability). tion. Emission spectra, photoluminescence quantum yields and fluorescence lifetimes (Table 3 and Fig. 6) demonstrate the significance of the aromatic substituent for room temperature fluorescence. Fluorescence wavelengths were additionally calculated for selected species in vacuum using time-dependent density functional theory (TDDFT). The predicted values for the $0-0$ transition are shown in Table 4 together with the oscillator strengths for the vertical fluorescent de-excitation. The emission spectra of species $\mathbf{5 b}$ and $\mathbf{5 e}$ are predicted to be considerably blue-shifted as compared to $\mathbf{5 f}, \mathbf{5 h}, \mathbf{5 i}$, and $\mathbf{5 j}$. However, their

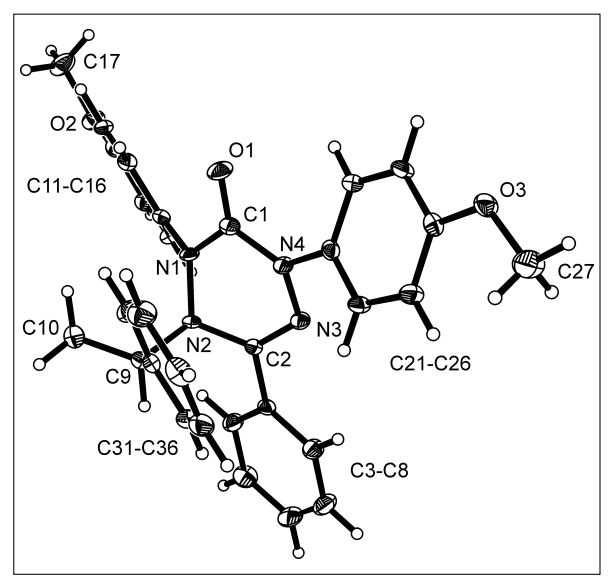

Fig. 5. X-ray crystal structure analysis of aryl substituted trapping product $\mathbf{5 i}$ (thermal ellipsoids are shown with $50 \%$ probability).

negligible oscillator strength explains why no emission could be detected for trapping products that do not bear a third aryl substituent (see also Table 3). As an example, the oscillator strength is five times higher for $\mathbf{5 i}$ than for $\mathbf{5 e}$, explaining its comparably high quantum yield. The low intensity of $\mathbf{5 b}$ and $\mathbf{5 e}$ can be understood in terms of the frontier orbitals (HOMO and LUMO) participating in the electronic transition. The HOMO mainly involves the heterocycle and two aromatic moieties, whereas the LUMO has significant delocalization on the third aromatic moiety (Fig. 7) that confers a push-pull character to the excited state. The TMS-acetylene substituted derivative $\mathbf{5 f}$ is an exception, as it does not have a phenyl substituent but still shows room temperature fluorescence, owing to the electron-rich TMS group, which introduces a slight LUMO delocalization. To corroborate this view, we investigated the effect of the silicon on the photophysical properties and treated $\mathbf{5 f}$ with $\mathrm{KF}$ to give the TMS-deprotected acetylene $\mathbf{5 n}$ in $70 \%$ yield (Scheme 1).

Not surprisingly, no emission was detectable at room temperature for $\mathbf{5 n}$. The predicted emission wavelengths for the aryl-substituted derivatives $\mathbf{5 i}, \mathbf{5 h}$, and $\mathbf{5 j}$ nicely coincide with the measured wavelengths in frozen matrices at $77 \mathrm{~K}$. We observe an emission red-shift upon HOMO destabilization by the methoxy groups $(\mathbf{5} \mathbf{h}$, 5i), and a blue-shift upon LUMO destabilization $(\mathbf{5 h}, \mathbf{5 j}, \mathbf{5 k})$. The fluorine atom, on the other hand, stabilizes the LUMO causing a bathochromic shift $(\mathbf{5 l})$. At $77 \mathrm{~K}$, the solvent molecules cannot rearrange their orientation to accommodate to the polarity changes upon optical excitation. At room temperature, however, the measured emission wavelengths show a slightly different trend, as the solvent dipoles can fit the changing polarity of the molecules. For instance, $\mathbf{5 i}$ and $\mathbf{5 j}$ are red-shifted as compared to $77 \mathrm{~K}$, while $\mathbf{5 h}$ appears blue- 
Table 3. Photophysical data of trapping products $5 a-5$ and $5 n$.

\begin{tabular}{|c|c|c|c|c|c|c|c|c|c|c|c|c|}
\hline & $\begin{array}{c}\lambda_{\text {abs }} \\
{[\mathrm{nm}]}\end{array}$ & $\begin{array}{c}\lambda_{\mathrm{exc}} \\
{[\mathrm{nm}]}\end{array}$ & $\begin{array}{c}\lambda_{\mathrm{em}} \\
{[\mathrm{nm}]}\end{array}$ & $\begin{array}{c}\tau^{\mathrm{a}} \\
{[\mathrm{ns}]}\end{array}$ & $\begin{array}{c}\Phi \\
{[\%]}\end{array}$ & $\begin{array}{c}\lambda_{\text {exc }} \\
{[\mathrm{nm}]}\end{array}$ & $\begin{array}{c}\lambda_{\mathrm{em}} \\
{[\mathrm{nm}]}\end{array}$ & $\begin{array}{c}\tau^{\mathrm{a}} \\
{[\mathrm{ns}]}\end{array}$ & $\begin{array}{c}\lambda_{\mathrm{exc}} \\
{[\mathrm{nm}]}\end{array}$ & $\begin{array}{c}\lambda_{\mathrm{em}} \\
{[\mathrm{nm}]}\end{array}$ & $\begin{array}{c}\tau^{\mathrm{a}} \\
{[\mathrm{ns}]}\end{array}$ & $\begin{array}{c}\Phi \\
{[\%]}\end{array}$ \\
\hline & \multicolumn{5}{|c|}{ room temperature } & \multicolumn{3}{|c|}{$77 \mathrm{~K}$} & \multicolumn{4}{|c|}{ solid state } \\
\hline $5 a$ & 257 & - & - & - & $<0.5$ & 300 & 415 & - & - & - & - & $<0.5$ \\
\hline $5 \mathbf{b}$ & 255 & - & - & - & $<0.5$ & 300 & 426 & 3.825 & - & - & - & $<0.5$ \\
\hline $5 c$ & 256 & - & - & - & $<0.5$ & 270 & 378 & 4.796 & - & - & - & $<0.5$ \\
\hline $5 d$ & 256 & - & - & - & $<0.5$ & 300 & 378 & 0.180 & - & - & - & $<0.5$ \\
\hline $5 e$ & 258 & - & - & - & $<0.5$ & 273 & 433 & 33.237 & - & - & - & $<0.5$ \\
\hline $5 f$ & 259 & 278 & 473 & 0.104 & $<0.5$ & 348 & 442 & 29.067 & - & - & . & $<0.5$ \\
\hline $5 g$ & 258 & - & - & - & $<0.5$ & 277 & 423 & 9.574 & - & - & - & $<0.5$ \\
\hline $5 \mathrm{~h}$ & 264 & 269 & 377 & 2.110 & 0.9 & 277 & 423 & 16.224 & 294 & 441 & 2.0523 & 2.6 \\
\hline $5 i$ & 255 & 250 & 486 & 0.590 & 1.4 & 285 & 438 & 22.395 & 300 & 434 & 2.448 & 13.7 \\
\hline $5 \mathbf{j}$ & 268 & 277 & 442 & 0.395 & $<0.5$ & 336 & 414 & 12.595 & 324 & 409 & 1.984 & 13.0 \\
\hline $5 k$ & 341 & 261 & 404 & 0.091 & $<0.5$ & 270 & 406 & 3.550 & 285 & 423 & 0.097 & 1.1 \\
\hline 51 & 259 & 341 & 420 & 0.820 & $<0.5$ & 275 & 452 & 17.485 & 295 & 415 & 2.488 & 12.9 \\
\hline $5 n$ & 255 & - & - & - & $<0.5$ & 269 & 444 & 17.427 & - & - & - & $<0.5$ \\
\hline
\end{tabular}

antensity weighted average lifetime

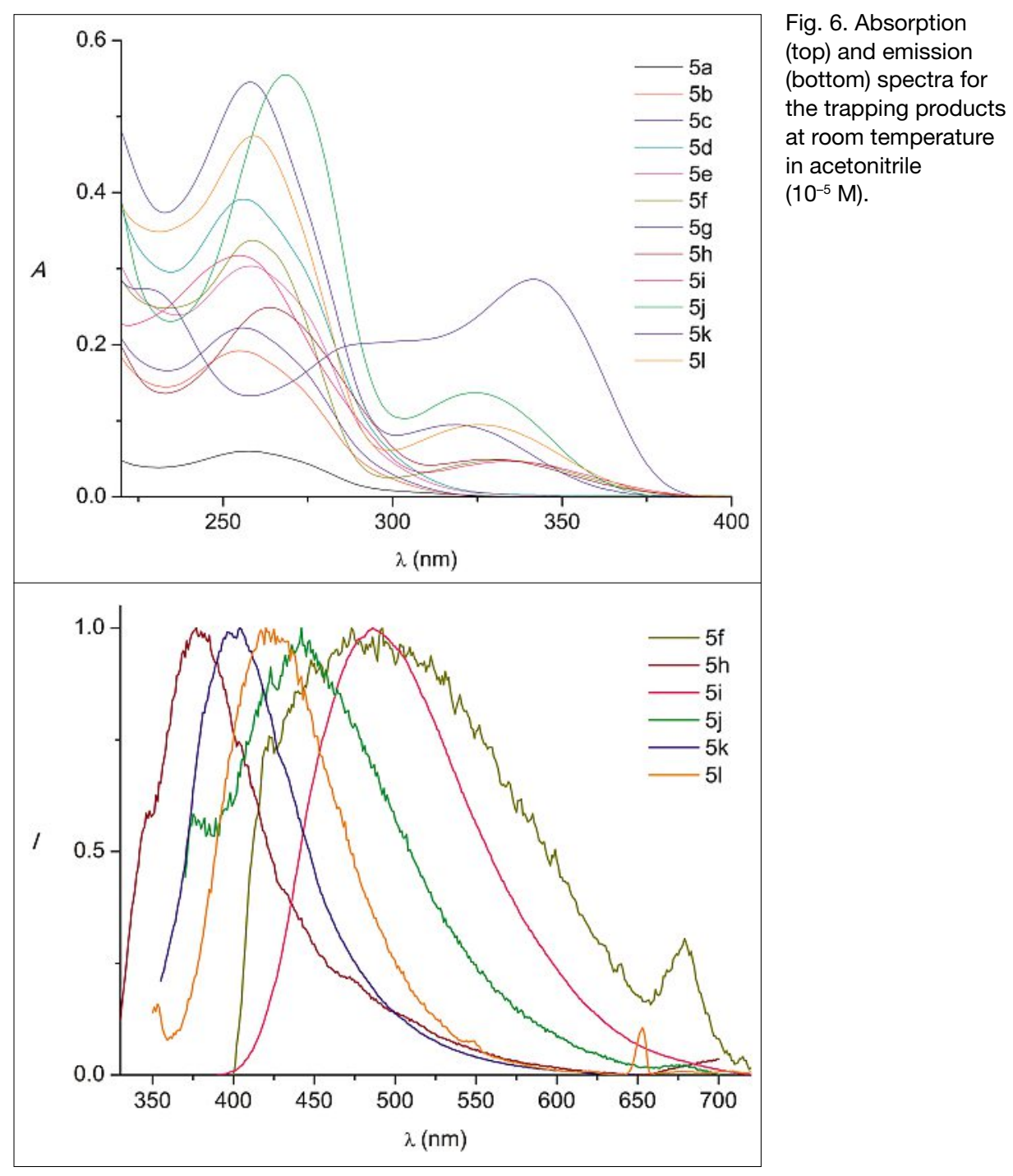

shifted, thus falling between $\mathbf{5 i}$ and $\mathbf{5 j}$ in fluid solution. This can be attributed to the fact that the threefold methoxy-substituted derivative $\mathbf{5 h}$ probably has a ground state with higher polarity than its excited state, leading to a blue-shifted emission in polar
Table 4. Calculated parameters in vacuum obtained from TDDFT/PBE0/6-31G* calculations: $\lambda_{\text {em }}$ is the $0-0$ emission wavelength in $\mathrm{nm}$ and $f$ is the oscillator strength for the vertical transition from the $\mathrm{S}_{1}$ minimum.

\begin{tabular}{|c|c|c|}
\hline & $\lambda_{\text {em }}$ & $f$ \\
\hline $\mathbf{5 b}$ & 360 & 0.0084 \\
\hline $\mathbf{5 e}$ & 346 & 0.0106 \\
\hline $\mathbf{5 f}$ & 436 & 0.0232 \\
\hline $\mathbf{5 h}$ & 428 & 0.0656 \\
\hline $\mathbf{5 i}$ & 447 & 0.0556 \\
\hline $\mathbf{5 j}$ & 396 & 0.0710 \\
\hline
\end{tabular}

solvents. $5 \mathbf{i}$ and $\mathbf{5 j}$ on the other hand are less symmetric and exhibit a more polar excited state compared with the ground state (more push-pull character), thus leading to red-shifted emission in solution.

In addition to the static TDDFT calculations, we have also performed excited state Car-Parrinello molecular dynamics simulations for species $\mathbf{5 a}, \mathbf{5 b}$, and $\mathbf{5 f}$ to investigate their stability. Although the simulations were carried out at an elevated temperature to account for the energy release during geometric relaxation from the Franck-Condon region to the $\mathrm{S}_{1}$ minimum, there are clear indications that these molecules are prone to fragmentation due to $\mathrm{C}-\mathrm{N}$ homolysis and formation of a styryl radical. This is experimentally corroborated by the study of compound $\mathbf{5 e}$, which slowly decomposes to verdazyl radical $4 \mathbf{e}$ in the presence of light. 


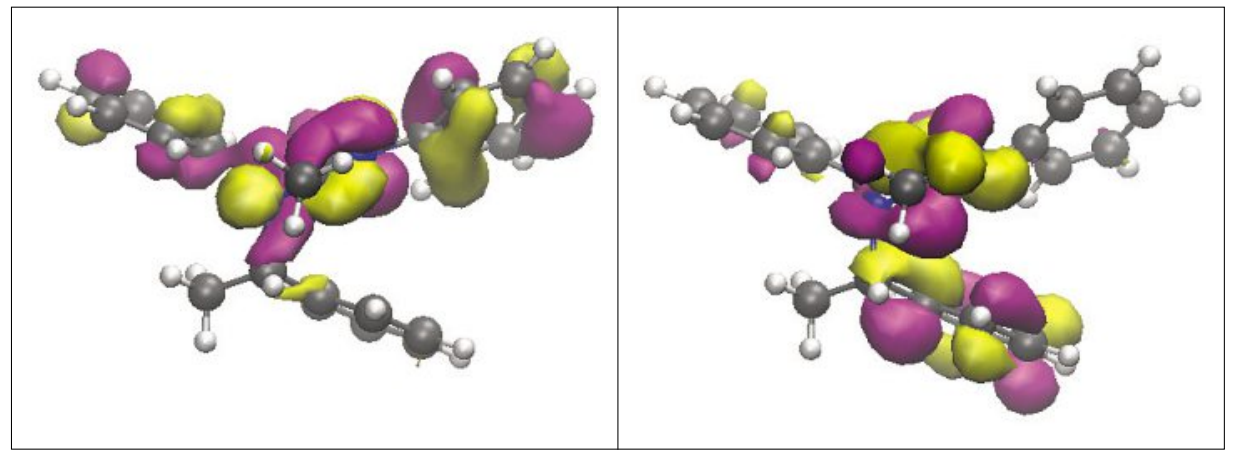

Fig. 7. HOMO (left) and LUMO (right) of $5 \mathbf{b}$.

每

Scheme 1. Synthesis of the TMSdeprotected acetylene $5 n$.

\section{Conclusions}

In summary, we presented the synthesis and characterization of various substituted 1,5-diphenyl-6-oxo verdazyl radicals and their use as potential fluorescent spin sensors. As pointed out, the $\mathrm{C}(3)$-substituent clearly is substantial for the profluorescent behavior. Spectroscopic measurements demonstrate the significance of the aryl substituent at the C(3)-position for room temperature fluorescence, as it enhances the delocalization of the LUMO. Interestingly, the emission wavelength can be tuned by selective destabilization of the HOMO and the LUMO.
[3] A. Yang, T. Kasahara, E. K. Y. Chen, G. K. Hamer, M. K. Georges, Eur. J. Org. Chem. 2008, 4571.

[4] L. J. Berliner, 'Spin Labeling: Theory and Application', vol. 2, Academix Press, New York, 1979.

[5] E. K. Y. Chen, D. Chan-Seng, P. O. Otieno, R. G. Hicks, M. K. Georges, Macromolecules 2007, 40, 8609 .

[6] D. Matuschek, S. Eusterwiemann, L. Stegemann, C. Doerenkamp, B. Wibbeling, C. G. Daniliuc, N. L. Doltsinis, C. A. Strassert, H. Eckert, A. Studer, Chem. Sci. 2015, 6, 4712.

[7] C. Chatgilialoglu, A. Studer, 'Encyclopedia of Radicals in Chemistry, Biology and Materials', John Wiley and Sons, Chichester, 2012.

[8] a) J. P. Blinco, K. E. Fairfull-Smith, B. J. Morrow, S. E. Bottle, Aust. J. Chem. 2011, 64, 373; b) R. Braslau, F. Rivera III, E. Lilie, M. E. Cottman, J. Org. Chem. 2013, 78, 238.

[9] B. A. Chalmers, S. Saha, T. Nguyen, J. McMurtrie, S. T. Sigurdsson, S. E. Bottle, K.-S. Masters, Org. Lett. 2014, 16, 5528.

[10] H-Y. Ahn, K. E. Fairfull-Smith, B. J. Morrow, V. Lussini, B. Kim, M. V. Bondar, S. E. Bottle, K. D. Belfield, J. Am. Chem. Soc. 2012, 134, 4721.

[11] K. E. Fairfull-Smith, J. P. Blinco, D. J. Keddie, G. A. George, S. E. Bottle, Macromolecules 2008, 41, 1577.

[12] a) R. Milcent, G. Barbier, F. Mazouz, K. B. Aziza, France Pat. 1989, WO/1989/004309; b) R. Milcent, G. Barbier, S. Capelle, J.-P. Catteau, J. Heterocycl. Chem. 1994, 31, 319; c) Y. Masuda, M. Kuratsu, S. Suzuki, M. Kozaki, D. Shiomi, K. Sato, T. Takui, K. Okada, Polyhedron 2009, 28, 1950.

[13] K. Matyjaszewski, B. E. Woodworth, X. Zhang, S. G. Gaynor, Z. Metzner, Macromolecules 1998, 31, 5955.

[14] a) L. S. Degtyarev, Y. I. Gorlov, Zh. Strukt. Khim. 1975, 16, 769; b) F. A. Neugebauer, H. Fischer, C. Krieger, J. Chem. Soc., Perkin Trans. 1 1993, 2, 535; c) R. G. Hicks, R. Hooper, Inorg. Chem. 1999, 38, 284; d) C. L. Barr, P. A. Chase, R. G. Hicks, M. T. Lemaire, C. L. Stevens, J. Org. Chem. 1999, 64, 8893; e) M. Chahma, K. Macnamara, A. Van der Est, A. Alberola, V. Polo, M. Pilkington, New J. Chem. 2007, 31, 1973; f) J. B. Gilroy, S. D. J. McKinnon, P. Kennepohl, M. S. Zsombor, M. J. Ferguson, L. K. Thompson, R. G. Hicks, J. Org. Chem. 2007, 72,8062 . 